\title{
Effect of dopant atoms on the roughness of III-V semiconductor cleavage surfaces
}

\author{
P. Quadbeck, Ph. Ebert, ${ }^{\text {a) }}$ and K. Urban \\ Institut für Festkörperforschung, Forschungszentrum Jülich GmbH, 52425 Jülich, Germany \\ J. Gebauer and R. Krause-Rehberg \\ FB Physik, Martin-Luther-Universität Halle, 06099 Halle, Germany
}

(Received 13 September 1999; accepted for publication 15 November 1999)

\begin{abstract}
We demonstrate that the presence of dopant atoms influences the roughness, morphology, and optical mirror properties of III-V semiconductor (110) cleavage surfaces. High concentrations of Te dopant atoms in GaAs lead to macroscopically curvatured (110) cleavage surfaces with high step concentrations. This "glass-like" fracture behavior is explained by the "lattice superdilation phenomenon" induced by high concentrations of Te dopant atoms in GaAs. () 2000 American Institute of Physics. [S0003-6951(00)02303-2]
\end{abstract}

Cleavage of III-V compound semiconductors in the zinc blende structure yield nearly perfect and atomically flat (110) surfaces. Such cleavage surfaces are well suited to be used as mirror planes of optical resonators in III-V semiconductor laser diodes. ${ }^{1}$ However, the cleavage of III-V semiconductors is a very delicate process. For example, it has been demonstrated that dynamical instabilities of the fracture process can lead to rough surfaces. ${ }^{2}$ Such low quality cleavage surfaces can severely limit the properties of optical resonators in semiconductor lasers and thus reduce the intensity of the laser itself. Therefore the ability to produce perfect cleavage surfaces, and naturally the understanding of the factors influencing the fracture process, is essential for the fabrication of high quality optical resonators.

In this letter we demonstrate that the presence of dopant atoms influences the roughness and morphology of III-V semiconductor cleavage surfaces on the atomic as well as macroscopic scale. We show that the lattice superdilation phenomenon' induced by high concentrations of Te dopant atom in $\mathrm{GaAs}^{3}$ affects the fracture process such that very rough (110) cleavage surfaces are produced. These surfaces exhibit even a "glass-like" fracture on the macroscopic scale making them unusable for mirrors in optical resonators.

In order to determine the influence of dopant atoms on the roughness of cleavage surfaces, we investigated the cleavage properties of GaAs, GaP, and InP single crystals doped with $\mathrm{Zn}, \mathrm{Cd}, \mathrm{Si}, \mathrm{S}, \mathrm{Sn}$, and Te. Each sample was well oriented along the [110] direction and we cut two cleavage slots into opposite sides along the oriented long axis. We cleaved the samples using a double wedge technique in $\langle 001\rangle$ and $\langle 110\rangle$ directions. Both cleavage directions yield similar results. All other cleavage directions were avoided, in order to exclude any possible influence of the orientation on the resulting surface morphology. The surfaces were investigated on the atomic scale by scanning tunneling microscopy (STM) and macroscopically by light optical microscopy.

On the macroscopic scale typical (110) cleavage sur-

\footnotetext{
a) Author to whom correspondence should be addressed; electronic mail: p.ebert@fz-juelich.de
}

faces of III-V semiconductors are mirror-like flat. Sometimes macroscopic cleavage steps occur, but the different macroscopic terraces of the surfaces have all the same orientation and no curvature at all. In contrast to this well known and characteristic picture, surfaces obtained by cleavage of highly Te doped GaAs $\left(5 \times 10^{18} \mathrm{~cm}^{-3}\right)$ exhibit a pronounced curvature (Fig. 1), although exactly the same cleavage setup and procedures were used. The light reflections on the cleavage surface shown in Fig. 1 visualize the large curvature of the cleavage surface of Te doped GaAs. We observed height differences of $0.1 \mathrm{~mm}$ over distances of $2 \mathrm{~mm}$. Figure 1 demonstrates that the fracture is more glass-like, but not that expected for the cleavage of a material with perfect cleavage planes.

Figure 2 shows STM images of the atomic-scale morphology of different cleavage surfaces. All surfaces investigated (more than 20 samples) except those of highly $\mathrm{Te}$ doped GaAs (two samples) have a relatively small density of steps and wide atomically flat terraces. This observation is independent of the material ( $\mathrm{GaAs}, \mathrm{InP}, \mathrm{GaP})$ and of the doping ( $n$ as well as $p$ doping). We could not detect any changes of the steps density for different doping concentra-

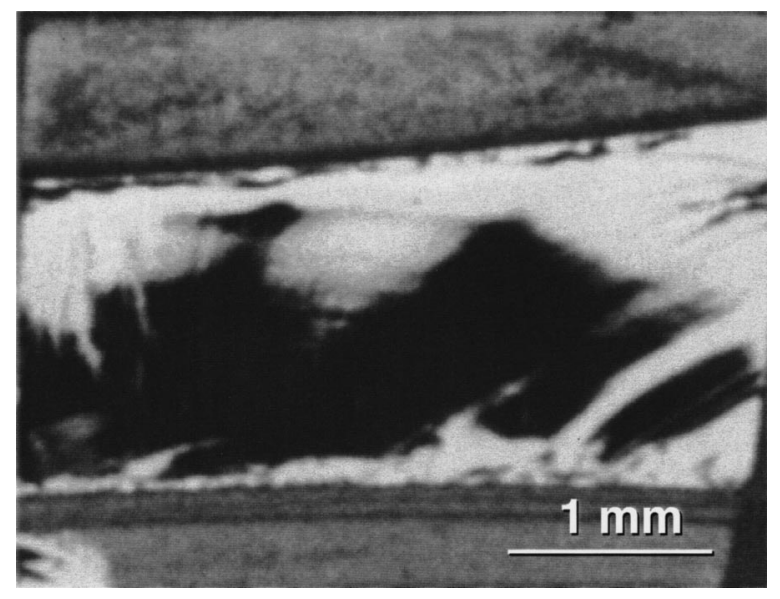

FIG. 1. Photograph of the light reflections on a nominally (110) cleavage surface of $5 \times 10^{18} \mathrm{~cm}^{-3}$ Te doped GaAs. The light reflections demonstrate the curvature of the surface. 

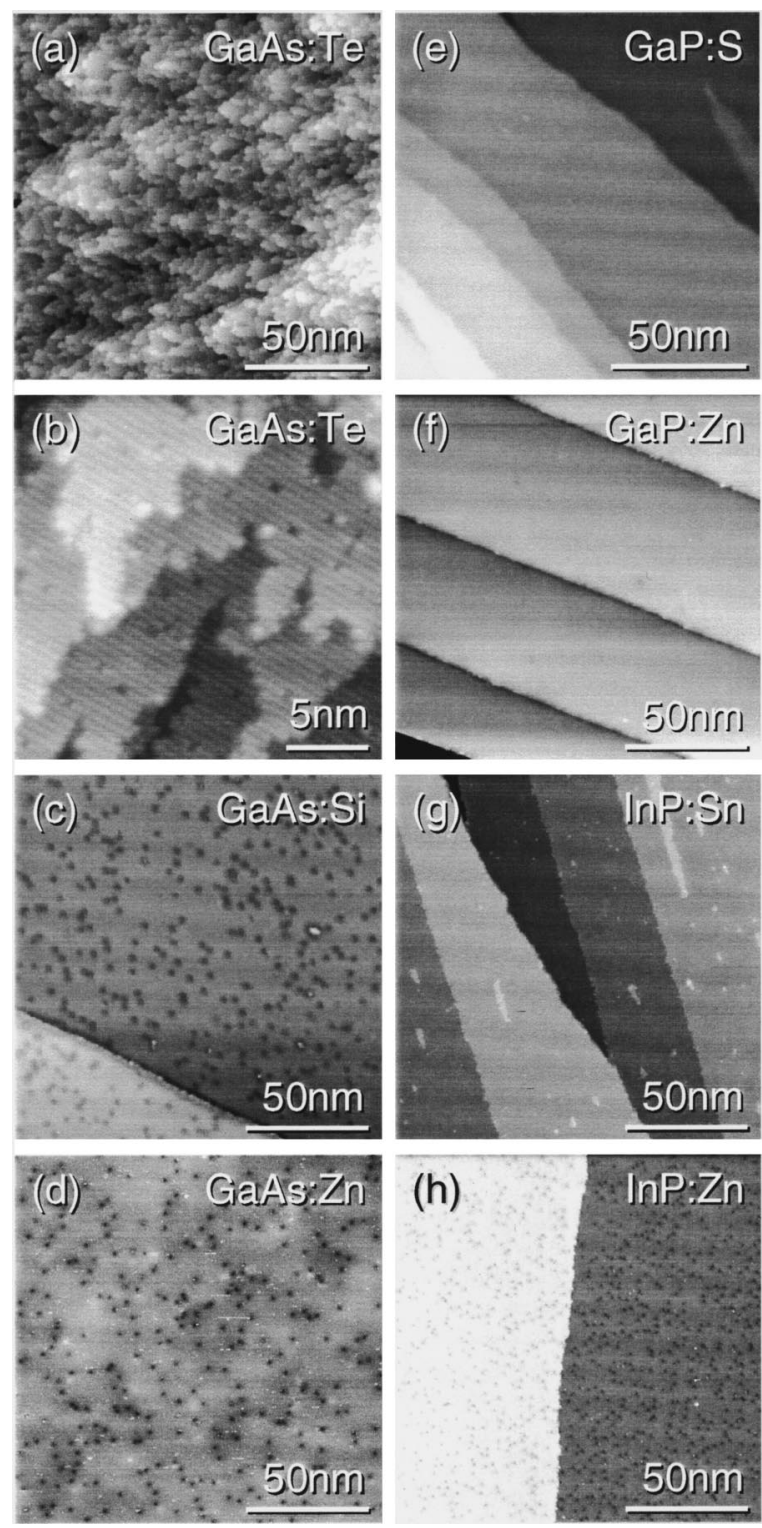

FIG. 2. Overview of the morphology and roughness induced by steps on (a) and (b) $5 \times 10^{18} \mathrm{~cm}^{-3}$ Te doped GaAs, (c) $1.3 \times 10^{19} \mathrm{~cm}^{-3} \mathrm{Si}$ doped GaAs, (d) $2 \times 10^{17} \mathrm{~cm}^{-3} \mathrm{Zn}$ doped GaAs, (e) $(5.6-6.0) \times 10^{17} \mathrm{~cm}^{-3} \mathrm{~S}$ doped GaP, (f) $(1.7-5.8) \times 10^{17} \mathrm{~cm}^{-3} \mathrm{~S}$ doped GaP, (g) $(0.9-1.8) \times 10^{18} \mathrm{~cm}^{-3}$ Sn doped InP, and (h) $(1.3-2.1) \times 10^{18} \mathrm{~cm}^{-3} \mathrm{Zn}$ doped InP (110) surfaces. Cleavage surfaces of Te doped GaAs have very high step concentrations.

tions of $\mathrm{Zn}$ or $\mathrm{Cd}$ doped $\operatorname{InP}\left(5 \times 10^{17}-8 \times 10^{18} \mathrm{~cm}^{-3}\right)$ and $\mathrm{Si}$ or $\mathrm{Zn}$ doped GaAs $\left(2 \times 10^{17}-2.5 \times 10^{20} \mathrm{~cm}^{-3}\right)$. Only Te doped GaAs has an extremely high density of steps shown in Figs. 2(a) and 2(b).

High resolution STM images [Fig. 2(b)] demonstrate that, in addition, the step edges on Te doped GaAs are very rough with no preferred edge orientations. These observations are in contrast to those on all other surfaces, where we found smooth and straight steps, mostly one atomic layer high with well defined facets. Only in areas directly at macroscopic steps, we observed a high density of steps. However, still preferred and distinct edge orientations are observed and have been reported. ${ }^{4-6}$

Quantitative values of the step concentrations observed on differently doped GaAs, InP, and GaP (110) surfaces are shown in Fig. 3. The values are shown as a function of the difference of the covalent radii of the dopant and the host Downloaded 15 Dec 2006 to 134.94.122.39. Redistribution subject to AIP license or copyright, see http://apl.aip.org/apl/copyright.jsp

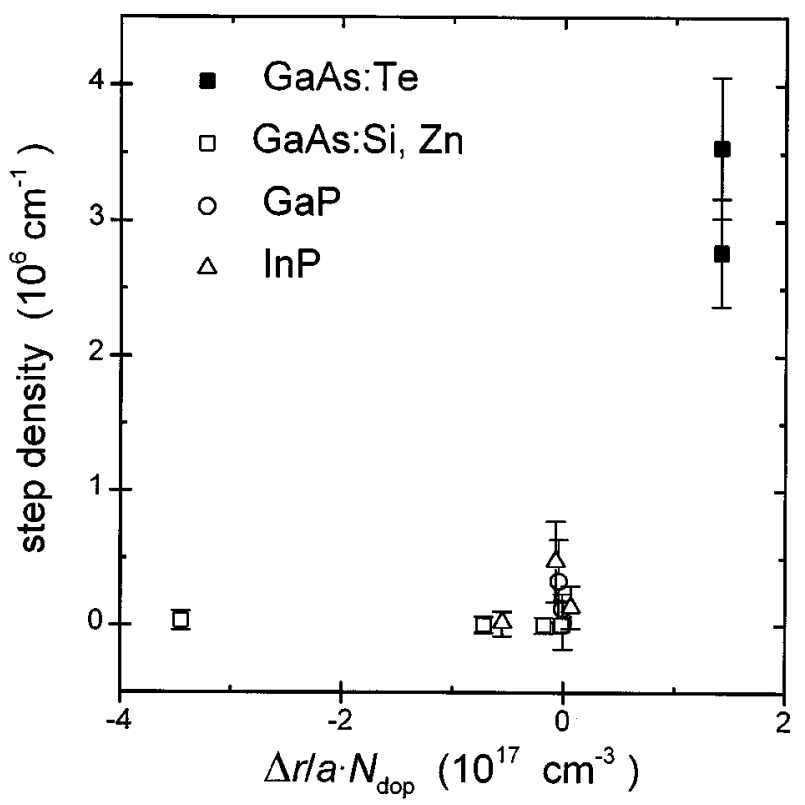

FIG. 3. Step concentrations on different III-V semiconductors observed in the STM images as a function of the difference in covalent radii between the dopant and host atoms $(\Delta r)$ normalized by the lattice constant $a$ and multiplied by the dopant concentration $N_{\text {dop }}$.

atoms $\Delta r$ normalized by the lattice constant $a$ and multiplied by the concentration of dopant atoms $N_{\text {dop }}$. This value is proportional to the total stress induced by the different radii of the dopant atoms (size effect). The two data points shown on the right side as filled squares represent the step concentrations on the Te doped GaAs (110) cleavage surfaces investigated. All other concentration values of GaAs (open circles), GaP (open squares), and InP (open triangles) (110) surfaces are much lower independent of the specific dopant concentration and the total stress based on the bare size effect of the dopant atom.

At this stage we discuss the origin of the different cleavage behavior of differently doped semiconductors. First we can exclude any misorientation of the samples to be responsible for the high step concentration, because first the samples are perfectly oriented within $\pm 0.5^{\circ}$ of the intended orientation, Second, samples miscut by several degrees still cleave exactly along the (110) plane yielding large atomically flat terraces. This preferred cleavage arises from the charge neutrality of the (110) planes, which consist of an equal number of anions and cations. We can also exclude point defects as origin for the different cleavage behavior observed, because crystals with high defect concentrations (highly Si doped ${ }^{7}$ and $\mathrm{Zn}$-diffused $\mathrm{GaAs}^{8}$ ) yielded atomically flat surfaces. Therefore we conclude that the dopant atoms affect the morphology of the cleavage surfaces of zinc blende structure III-V semiconductors.

If the dopant atoms affect the cleavage process we have to consider the stress induced by dopant atoms. It is well known that dopant atoms with different covalent radii than their host atoms introduce an average lattice dilation in semiconductors. ${ }^{3,9-11}$ This relative lattice dilation can be described according to Ref. 12 by

$$
\frac{\Delta a}{a}=\frac{\Delta r}{r} \cdot \frac{4}{\sqrt{3}} \cdot \frac{N_{\text {dop }}}{N_{\text {host }}}+\frac{D}{3 K} \cdot n,
$$




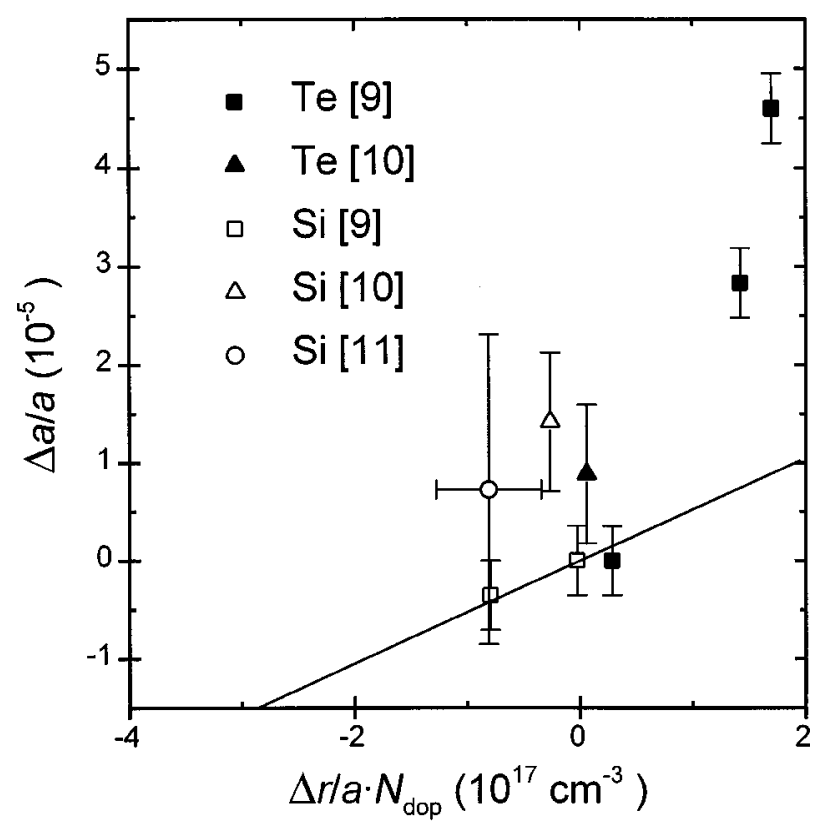

FIG. 4. Lattice dilation values measured in Refs. 9-11 as a function of the difference in covalent radii between the dopant and host atoms $(\Delta r)$ normalized by the lattice constant $a$ and multiplied by the dopant concentration $N_{\text {dop }}$. The solid line represents the calculated lattice dilation induced by the pure size effect of the differently sized dopant atom.

with $N_{\text {dop }}$ and $N_{\text {host }}$ being the concentrations of doping and host atoms, respectively. $n$ is the carrier concentration. The first term is the size effect of the dopant atom known as Vegard's law. ${ }^{13}$ The second term, usually small, describes the electronic contribution to the change of the lattice constant induced by the free charge carriers ( $D$ is the deformation potential and $K$ is the bulk modulus).

The lattice dilation values of $\mathrm{Si}$ and $\mathrm{Te}$ doped GaAs measured by $\mathrm{x}$-ray diffraction (Bond method) are shown in Fig. 4. Most of the data agree with the relative change of the lattice constant expected by the pure size effect or Vegard's law (solid line). However, the data points for highly $\mathrm{Te}$ doped GaAs are significantly higher than that expected by Vegard's law. This effect, called lattice superdilation, ${ }^{3}$ appears for Te doped GaAs above doping concentrations above about $3 \times 10^{18} \mathrm{~cm}^{-3}$ and disappears for low Te concentrations.

Above we already concluded that the dopant atoms affect the roughness of the cleavage planes. Here we find that the observed step concentrations on the cleavage surfaces and the measured lattice dilation values exhibit a clear similarity. Only for highly Te doped GaAs an unusually large lattice dilation is observed and a rough surface is formed by cleavage exactly for this type of material. This suggests that the lattice superdilation effect increases the roughness of the cleavage surfaces. This model can be tested best if a Te doped GaAs crystal is cleaved with a doping concentration below the critical one, where the superdilation effect starts. Indeed, in a recent STM investigation of GaAs doped with $5 \times 10^{17} \mathrm{~cm}^{-3} \mathrm{Te}$, only flat surfaces are reported. ${ }^{14}$

In conclusion, Te dopant atoms in high concentrations significantly increase the roughness and step concentrations on cleavage surfaces of GaAs, such that macroscopic curvatures can be observed. The influence of Te dopant atoms on the cleavage process is connected with the lattice superdilation phenomenon. The glass-like cleavage behavior observed limits the use of highly Te doped substrates for semiconductor laser diodes with cleaved mirror planes.

The authors thank K. H. Graf for technical support, G. Wassenhoven for preparation of Fig. 1, C. Domke and M. Simon for providing some STM images, and the Deutsche Forschungsgemeinschaft for financial support.

${ }^{1}$ J. Hech, The Laser Guidebook (McGraw-Hill, New York, 1986).

${ }^{2}$ K. Sauthoff, M. Wenderoth, A. J. Heinrich, M. A. Rosentreter, K. J. Engel, T. C. G. Reusch, and R. G. Ulbrich, Phys. Rev. B 60, 4789 (1999).

${ }^{3}$ J. B. Mullin, B. W. Straughan, C. M. H. Driscoll, and A. F. W. Willoughby, J. Appl. Phys. 47, 2584 (1976).

${ }^{4}$ Y.-N. Yang, B. M. Trafas, R. L. Siefert, and J. H. Weaver, Phys. Rev. B 44, 3218 (1991).

${ }^{5}$ R. Möller, R. Coenen, B. Koslowski, and M. Rauscher, Surf. Sci. 217, 289 (1989).

${ }^{6}$ M. Heinrich, C. Domke, Ph. Ebert, and K. Urban, Phys. Rev. B 53, 10894 (1996).

${ }^{7}$ C. Domke, Ph. Ebert, M. Heinrich, and K. Urban, Phys. Rev. B 54, 10288 (1996).

${ }^{8}$ M. Simon, thesis, RWTH Aachen, 1995.

${ }^{9}$ C. M. H. Driscoll, A. F. W. Willoughby, J. B. Mullin, and B. W. Straughan, Inst. Phys. Conf. Ser. 24, 275 (1975).

${ }^{10}$ J. F. C. Baker, M. Hart, M. A. G. Halliwell, and R. Heckingbottom, Solid-State Electron. 19, 331 (1976).

${ }^{11}$ P. F. Fewster and A. F. W. Willoughby, J. Cryst. Growth 50, 648 (1980).

${ }^{12}$ U. Pietsch and K. Unger, Phys. Status Solidi A 80, 165 (1983).

${ }^{13}$ L. Vegard, Z. Phys. 5, 17 (1921).

${ }^{14}$ A. Depuydt, C. Van Hasendonck, N. S. Maslova, V. I. Panov, S. V. Savinov, and P. I. Arseev, Phys. Rev. B 60, 2619 (1999). 